\title{
Modelos de ponto crítico para estimar danos causados pela ferrugem da folha da aveia branca
}

\author{
Erlei Melo Reis ${ }^{1}$, Ricardo Trezzi Casa ${ }^{2}$, Luiz Carlos Bevilaqua ${ }^{1}$
}

${ }^{1}$ Faculdade de Agronomia e Medicina Veterinária, Universidade de Passo Fundo, CP 611, CEP 99001-970, Passo Fundo, RS, erleireis@ tpo.com.br; ${ }^{2}$ Centro de Ciências de Agroveterinárias, Universidade do Estado de Santa Catarina, CP 281, 88520-000, Lages, SC, a2rtc@ cav.udesc.br Autor para correspondência: Erlei Melo Reis. erleireis@tpo.com.br Data de chegada: 22/08/2006. Aceito para publicação em: 09/04/2008

\section{RESUMO}

Reis, E.M.; Casa, R.T.; Bevilaqua, L.C. Modelo de ponto crítico para estimar danos causados pela ferrugem da folha da aveia branca. Summa Phytopathologica, v.34, n.3, p.238-241, 2008

Em experimentos conduzidos no campo, nas safras agrícolas de 1995 e 1996, gerou-se o gradiente da intensidade da ferrugem da folha da aveia branca, cultivar UPF 13, pela aplicação nos órgãos aéreos de doses crescentes do fungicida triadimenol. As equações das funções de dano foram obtidas pela correlação entre o rendimento de grãos e a incidência da doença em diferentes estádios fenológicos da cultura. $\mathrm{Na}$ safra de 1995 as equações obtidas foram $\mathrm{R}=2.103,5-17,983 \mathrm{I}$ e $\mathrm{R}=2.404,6-12,832 \mathrm{I}$, respectivamente para alongamento e emborrachamento, e em 1996, $R=3.889,2$ - 27,871I e $R=5.366,4$ -
20,999I, respectivamente para emborrachamento e floração ( $R=$ rendimento de grãos e I= incidência foliar). Estas equações, contendo o coeficiente de dano, permitem calcular o limiar de dano econômico (LDE) tomado como critério indicador do momento para o início do controle químico da ferrugem da folha da aveia. As reduções no rendimento de grãos, no peso do hectolitro e no peso de mil sementes, atingiram, respectivamente $57,13 \%, 16,64 \%$ e $21,49 \%$ na safra 1995 e $19,79 \%, 13,39 \%$ e $16,33 \%$, na safra 1996 .

Palavras-chave adicionais: controle químico, limiar de dano econômico, Puccinia coronata f.sp. avenae.

\section{ABSTRACT}

Reis, E.M.; Casa, R.T.; Bevilaqua, L.C. Critical yield models to estimate the damage caused by leaf rust in white oat. Summa Phytopathologica, v.34, n.3, p.238-241, 2008

In field experiments carried out in the 1995 and 1996 growing seasons, the gradient of leaf rust intensity on the white oat cultivar UPF 13 was generated by spraying the above ground plant parts of the crop with different rates of the fungicide triadimenol. Damage equations were obtained relating grain yield and disease incidence at different growing stages. In the 1995 growing season the equations were: $R=2,103.5-17.983 \mathrm{I}$ and $\mathrm{R}=2,404.6-12.832 \mathrm{I}$, for elongation and boot stage, respectively, and for $1996, \mathrm{R}=3,889.2-27.871 \mathrm{I}$ and
$\mathrm{R}=5,366.4-20.999 \mathrm{I}$ (where $\mathrm{R}=$ grain yield; $\mathrm{I}=$ disease as foliar incidence), for booting and flowering stages respectively. These equations, having the damage coefficient, may be used to calculate the economic damage threshold (LDE) as a criterion to indicate the moment for the fungicide application to control leaf rust in oats. Reductions in grain yield, hectoliter weight, and one thousand kernels weigh reached $57.13 \%, 16.64 \%$ and $21.49 \%$ respectively for the 1995 growing season and $19.79 \%, 13.39 \%$ and $16.33 \%$, for the 1996 season.

Additional keywords: chemical control, economic damage threshold, Puccinia coronata f.sp. avenae

A cultura da aveia branca (Avena sativa L.) ocupa posição de destaque na produção de grãos de cereais de inverno na Região Sul do Brasil, com cultivares de alto potencial de rendimento e qualidade de grãos. A cultura, no Brasil, por muito tempo teve pouca expressão, pois sua utilização visava, principalmente, a produção de forragens, isolada ou associada a outras espécies, cultivando-se principalmente, a aveia preta. Isto se atribuiu a falta de cultivares adaptadas às condições climáticas e aos danos causados principalmente pela ferrugem da folha, tendo como agente causal o fungo Puccinia coronata (Cda.) f.sp. avenae (Eriks) $(4,6)$.

A ferrugem da folha é considerada a doença mais destrutiva na aveia branca (2, 5). Segundo Martinelli et al. (10) na cultivar UFRGS 7 a ferrugem causou danos de até $50 \%$ no rendimento de grãos. A utilização de cultivares resistentes tem sido o principal método de controle da doença $(2,6)$. No entanto, o melhoramento genético não tem sido suficientemente efetivo no controle desta moléstia, considerando-se o período de tempo que as cultivares mantêm-se resistentes (6). Nas cultivares suscetíveis a ferrugem pode ser eficientemente controlada por fungicidas $(2,9)$. Forcelini et al. (3), avaliaram a eficiência do fungicida tebuconazole, no controle da ferrugem da folha, na cultivar UPF 13. O tratamento, a partir do surgimento das primeiras pústulas até $5 \%$ de severidade, reduziu a severidade final da doença em $80 \%$.

Muitos trabalhos discutem a necessidade e mostram a importância da quantificação de danos e perdas causadas pelas doenças de plantas $(1,7,8,18)$. Sem estimativas confiáveis das reduções de produção acarretadas pelos patógenos, como decidir, racionalmente, quando e quanto investir em controle? Ou ainda, como pode uma empresa, pública ou privada, investir na produção de variedades resistentes, em produtos químicos, em métodos alternativos de controle, sem ter 
conhecimento preciso da importância relativa da doença e dos benefícios correspondentes resultantes do seu controle? No Brasil, por exemplo, para a ferrugem da folha em trigo (14) e para o oídio em cevada (16) existem informações sobre a quantificação de danos. No caso da ferrugem da aveia são escassos os trabalhos publicados relacionando a intensidade da doença e danos causados que justifiquem o seu controle através de fungicidas os quais aumentam o custo de produção.

Os objetivos deste trabalho foram correlacionar a incidência da ferrugem da folha com o rendimento de grãos, obter a equação matemática de regressão da função de dano e calcular o limiar de dano econômico da doença em diferentes estádios fenológicos para servir de critério indicativo do momento para o controle químico econômico.

\section{MATERIAL E MÉTODOS}

Os ensaios foram conduzidos no campo experimental da Faculdade de Agronomia e Medicina Veterinária da Universidade de Passo Fundo em 1995 e 1996, em área com sistema plantio direto e rotação de culturas.

Sementes de aveia branca da cultivar UPF 13, suscetível à ferrugem da folha, foram previamente tratadas com o fungicida guazatina $(300 \mathrm{~g}$ de Panoctine 25\% PM para $100 \mathrm{~kg}$ de sementes), com o objetivo de controlar o fungo Drechslera avenae Eidam, visando impedir a transmissão do fungo para a parte aérea da cultura e interferir no estudo da ferrugem, a doença alvo do trabalho. A semeadura foi realizada no dia 23 de julho de 1995 e 12 de julho de 1996, utilizando-se uma semeadora de plantio direto para parcelas experimentais. Cada unidade experimental constou de uma parcela com 1,2m de largura por cinco metros de comprimento (sete linhas de semeadura espaçadas $20 \mathrm{~cm}$ ).

A densidade de plantas, a adubação de base e de cobertura, o manejo de pragas e plantas daninhas, foram realizadas conforme a recomendação de pesquisa para o cultivo de aveia (6).

No presente trabalho o fungicida triazol sistêmico triadimenol (Bayfidan $250 \mathrm{CE}$ ) foi usado como uma ferramenta de pesquisa para gerar o gradiente da ferrugem segundo proposto por Sah \& McKenzie (17). Para tal, foram usadas doses crescentes do fungicida (Tabelas 1 e 2). Os tratamentos com fungicida foram aplicados duas vezes, iniciando quando detectada a ferrugem utilizando o critério de incidência foliar e a segunda aos 20 dias após a primeira pulverização. A aplicação foi feita com pulverizador costal de precisão, com pressão gerada por $\mathrm{CO}_{2}$, com quatro pontas jato em leque XR 110-02. O delineamento experimental foi de blocos ao acaso, com sete tratamentos e quatro repetições.

A avaliação da evolução da doença foi feita nos estádios de alongamento, emborrachamento e floração, determinando-se a incidência foliar da doença nas folhas verdes e expandidas, de 10 plantas, coletadas, ao acaso, por parcela. Considerou-se infectada a folha que apresentou no mínimo uma pústula com esporulação visível a olho-nu.

A colheita foi feita mecanicamente com automotriz de parcelas. Calculou-se o rendimento em $\mathrm{kg}$ de grãos/ha, baseando-se na área experimental colhida. Determinou-se também o peso de mil sementes e o peso do hectolitro.

Os dados de incidência foliar da doença foram submetidos a análise de variância, com transformação em arc seno - arc seno [raiz (x/k)], onde x é a incidência e k é uma constante com valor igual a 100, e as

Tabela 1. Incidência da ferrugem da folha, peso de mil sementes (PMS), peso do hectolitro (PH) e rendimento de grãos de aveia cultivar UPF 13 na safra agrícola de 1995

\begin{tabular}{|c|c|c|c|c|c|c|}
\hline \multirow[t]{2}{*}{ Tratamentos $/$ Doses $^{1}$} & \multicolumn{2}{|c|}{ Incidência (\%) } & \multirow[t]{2}{*}{ PMS(g) } & \multirow[t]{2}{*}{$\mathbf{P H}\left(\mathrm{kg} \cdot \mathrm{hL} \mathbf{L}^{-1}\right)$} & \multirow[t]{2}{*}{ Rendimento $\left(\right.$ kg.ha $\left.^{-1}\right)$} & \multirow[t]{2}{*}{$\operatorname{Dano}(\%)$} \\
\hline & Elongação & Emborrachamento & & & & \\
\hline Testemunha & $63,3 \mathrm{a}$ & $96,6 \mathrm{a}$ & $25,2 \mathrm{c}$ & $50,1 \quad \mathrm{c}$ & $855 \mathrm{c}$ & 57,13 \\
\hline Triadimenol $62,5 \mathrm{~mL}$ & $47,7 \mathrm{ab}$ & $90,7 \mathrm{a}$ & $27,5 \mathrm{bc}$ & 53,4 bc & $1.313 \mathrm{bc}$ & 34,19 \\
\hline Triadimenol $125 \mathrm{~mL}$ & 28,3 bc & $72,2 \mathrm{ab}$ & $28,0 \mathrm{ab}$ & $56,8 \mathrm{ab}$ & $1.550 \mathrm{ab}$ & 22,32 \\
\hline Triadimenol $250 \mathrm{~mL}$ & $17,6 \mathrm{~cd}$ & $67,7 \mathrm{ab}$ & $30,5 \mathrm{ab}$ & $58,0 \mathrm{ab}$ & $1.744 \mathrm{ab}$ & 12,57 \\
\hline Triadimenol $500 \mathrm{~mL}$ & $16,6 \mathrm{~cd}$ & 53,4 bc & $30,2 \mathrm{ab}$ & $57,5 \mathrm{ab}$ & $1.908 \mathrm{a}$ & 4,34 \\
\hline Triadimenol $1000 \mathrm{~mL}$ & 8,3 & 27,7 & $30,5 \mathrm{ab}$ & $59,8 \mathrm{ab}$ & $1.925 \mathrm{a}$ & 3,48 \\
\hline Triadimenol $2000 \mathrm{~mL}$ & 5,2 & 23,6 & $32,1 \mathrm{a}$ & $60,1 \mathrm{a}$ & $1.995 \mathrm{a}$ & - \\
\hline C.V. $(\%)$ & 19,01 & 16,60 & 4,59 & 5,02 & 15,23 & \\
\hline
\end{tabular}

${ }^{1}$ Doses do produto comercial em $\mathrm{mL}$ por hectare; Médias seguidas da mesma letra na coluna não diferem entre si pelo teste de Tukey (P>0,05).

Tabela 2. Incidência da ferrugem da folha, peso de mil sementes (PMS), peso do hectolitro (PH) e rendimento de grãos de aveia cultivar UPF 13 na safra agrícola de 1996

\begin{tabular}{|c|c|c|c|c|c|c|}
\hline \multirow[t]{2}{*}{ Tratamentos /Doses ${ }^{1}$} & \multicolumn{2}{|c|}{ Incidência (\%) } & \multirow[t]{2}{*}{$\operatorname{PMS}(\mathrm{g})$} & \multirow[t]{2}{*}{$\operatorname{PH}\left(\mathrm{kg} \cdot \mathrm{hL} \mathrm{L}^{-1}\right)$} & \multirow[t]{2}{*}{ Rendimento $\left(\mathrm{kg} \cdot \mathrm{ha}^{-1}\right)$} & \multirow[t]{2}{*}{$\operatorname{Dano}(\%)$} \\
\hline & Emborrachamento & Floração & & & & \\
\hline Testemunha & $28,3 \mathrm{a}$ & $99,0 \mathrm{a}$ & $29,2 \quad d$ & $48,5 \quad \mathrm{~d}$ & $3.120 \mathrm{a}$ & 19,79 \\
\hline Triadimenol $62,5 \mathrm{~mL}$ & $22,4 \mathrm{a}$ & 99,1 a & $30,6 \mathrm{~cd}$ & $50,4 \mathrm{~cd}$ & $3.187 \mathrm{a}$ & 18,07 \\
\hline Triadimenol $125 \mathrm{~mL}$ & $24,1 \mathrm{a}$ & 96,7 a & $30,2 \quad d$ & 51,6 bc & $3.294 \mathrm{a}$ & 15,32 \\
\hline Triadimenol $250 \mathrm{~mL}$ & $9,1 \mathrm{a}$ & $98,2 \mathrm{a}$ & 31,7 bcd & $51,6 \mathrm{bc}$ & $3.494 \mathrm{a}$ & 10,17 \\
\hline Triadimenol $500 \mathrm{~mL}$ & $4,9 \mathrm{a}$ & 76,5 a & $33,4 a b c$ & $53,8 \mathrm{ab}$ & $3.600 \mathrm{a}$ & 7,45 \\
\hline Triadimenol $1000 \mathrm{~mL}$ & $7,5 \mathrm{a}$ & 87,5 a & $33,9 \mathrm{ab}$ & 54,9 a & $3.786 \mathrm{a}$ & 2,67 \\
\hline Triadimenol $2000 \mathrm{~mL}$ & 9,9 a & $71,3 \mathrm{a}$ & 34,9 a & $56,0 \mathrm{a}$ & $3.890 \mathrm{a}$ & - \\
\hline C.V. $(\%)$ & 55,48 & 19,53 & 3,79 & 2,24 & 7,12 & \\
\hline
\end{tabular}

${ }^{1}$ Doses do produto comercial em $\mathrm{mL}$ por hectare; Médias seguidas da mesma letra na coluna não diferem entre si pelo teste de Tukey (P>0,05). 
Tabela 3. Funções de dano (normais e transformadas para $1.000 \mathrm{~kg}$ ) para a ferrugem da folha da aveia nos anos de 1995 e 1996 em função de estádios fenológicos

\begin{tabular}{|c|c|c|c|c|}
\hline Ano & Estádiofenológico & Equação & Coeficiente de Determinação $\left(\mathbf{R}^{2}\right)$ & $\operatorname{Significância}(\mathbf{p}=)$ \\
\hline \multirow{3}{*}{1995} & Alongamento & $R=2.103-17,983$ I $R=1.000-8,55 \mathrm{I}$ & 0,98 & 0,000002 \\
\hline & Emborrachamento & $R=2.404,6-12,832 \mathrm{IR}=1.000-5,34 \mathrm{I}$ & 0,79 & 0,007 \\
\hline & Emborrachamento & $\mathrm{R}=3.889,2-27,871 \mathrm{IR}=.1000-7,17 \mathrm{I}$ & 0,73 & 0,014 \\
\hline 1996 & Floração & $R=5.366,4-20,999 \mathrm{IR}=1.000-3,91$ & 0,68 & 0,022 \\
\hline
\end{tabular}

$\mathrm{R}=$ Rendimento de grãos em kg.ha ${ }^{-1}$; I= Incidência foliar da ferrugem.

médias comparadas pelo teste de Tukey a $5 \%$ de probabilidade. Procedeu-se a análise de regressão entre a incidência foliar da doença, variável independente, e o rendimento de grãos, variável dependente, para cada estádio de crescimento da cultura, obtendo-se as equações das funções de dano e o coeficiente de dano $(\mathrm{Cd})$ para uso no cálculo do limiar de dano econômico $(13,15)$.

\section{RESULTADOS E DISCUSSÃO}

Com a metodologia usada (17) gerou-se o gradiente da doença e do rendimento de grãos correspondente nos estádios de elongação e de emborrachamento na safra de 1995 (Tabela 1) e nos estádios de emborrachamento e floração na safra de 1996 (Tabela 2). Houveram efeitos significativos dos tratamentos sobre o controle da doença, peso do hectolitro $(\mathrm{PH})$, peso de mil sementes (PMS) e no rendimento de grãos (Tabelas 1 e 2), principalmente na safra de 1995.

Em 1996, as condições climáticas ocorrentes, durante o desenvolvimento da cultura, não foram tão favoráveis à epidemia da doença como na safra anterior. Por conseguinte, a ferrugem ocorreu mais tarde do que no ano anterior, de maneira que o gradiente da doença foi gerado apenas nos estádios fenológicos do final da cultura. Por isso, na safra 1995 o dano foi de 57,13\%, enquanto na safra 1996 o dano foi de $19,79 \%$.

A diferença no rendimento de grãos entre as safras deve-se ao clima mais favorável a doença em 1995 e mais favorável à cultura em 1996. Correspondentes as mesmas safras o dano no PH foi de $16,64 \%$, no primeiro experimento e $13,39 \%$ no segundo e o dano no PMS de 21,49 no primeiro e $16,33 \%$, respectivamente.

As equações das funções de dano obtidas pela correlação entre o rendimento de grãos e a incidência foliar da doença constam na Tabela 3. O efeito da intensidade da ferrugem da folha da aveia sobre o rendimento $\left(\mathrm{kg} \cdot \mathrm{ha}^{-1}\right)$ determinou uma redução na quantidade de grãos de até $57,13 \%$ (Tabela 1). Este valor é semelhante ao obtido por outros autores que trabalharam com a cultivar UPF 5 tendo obtido dano de 50\%, correspondendo a uma severidade foliar de $90 \%$ (10).

As funções de dano geradas neste trabalho (Tabela 3) podem ser usadas no cálculo do limiar de dano econômico (LDE). Para a determinação LDE toma-se como base a equação de Munford \& Norton (11) desenvolvida para a quantificação do LDE para nematóides e no presente trabalho aplicada à doenças: $\mathrm{ID}=(\mathrm{Cc} / \mathrm{Pp} \times \mathrm{Cd}) \times \mathrm{Ec}$ onde $\mathrm{ID}=$ Intensidade da doença (Incidência foliar da ferrugem da folha); $\mathrm{Cc}=$ Custo do controle (12) (fungicida, combustível, amassamento, salário operador) de $\mathrm{R} \$ 90,00 ; \mathrm{Pp}=$ Preço da tonelada de grãos de aveia (base de cálculo de R $\$ 360,00$, Cooperativa Ibirubá - RS, em 01/ 04/08); $\mathrm{Cd}=$ Coeficiente de dano (obtido da equação da função de dano para a elongação: $R=1.000-8,55 \mathrm{I} ; \mathrm{R}^{2}=0,98 ; \mathrm{p}=0,000002$ da Tabela 3); e Ec= Eficiência de controle de $73,8 \%$ foi calculada com dados da Tabela 1 , correspondente ao estádio de alongamento (diferença entre incidência da testemunha e a da dose $(500 \mathrm{~mL})$ recomendada para o controle da doença em lavouras comerciais. Neste caso, para cada $1 \%$
Quadro 1. Exemplo do cálculo do limiar de dano econômico para o estádio de alongamento na cultura da aveia cultivar com reação 'a ferrugem semelhante à UPF 13

\begin{tabular}{ll}
\hline $\mathrm{ID}=(\mathrm{Cc} / \mathrm{Pp} \times \mathrm{Cd}) \times \mathrm{Ec}$ & Valor das Variáveis \\
$\mathrm{ID}=(52,50 / 192,50 \times 0,03326) \times 0,74$ & $\mathrm{Cc}=\mathrm{R} \$ 52,50$ \\
$\mathrm{ID}=(52,50 / 6,40) \times 0,74$ & $\mathrm{Pp}=\mathrm{R} \$ 192,50$ \\
$\mathrm{ID}=8,2 \times 0,74$ & $\mathrm{Cd}=33,26$, ou $0,03326 \mathrm{t}$. \\
$\mathrm{ID}=6,07$ & $\mathrm{Ec}=73,8 \%$ ou 0,74 \\
$\mathrm{ID}=\mathrm{LDE}$ ou $6,07 \%$ de Incidência & \\
\hline
\end{tabular}

$\overline{\mathrm{ID}}=$ intensidade da doença; $\mathrm{Cc}=$ custo do controle; $\mathrm{Pp}=$ preço da tonelada da aveia; $\mathrm{Cd}=$ coeficiente de dano; e Ec = eficiência do controle.

de incidência da doença ocorreu redução de $8,55 \mathrm{~kg} \cdot \mathrm{ha}^{-1}$ no estádio de alongamento, para cada $1.000 \mathrm{~kg}$ de grãos de aveia produzidos.

Na prática o valor do Cd deve ser ajustado para o rendimento potencial da lavoura de aveia, aonde a ferrugem deve ser controlada. Por exemplo, $3.890 \mathrm{~kg} \cdot \mathrm{ha}^{-1}$ (Tabela 2, tratamento com $2.000 \mathrm{~mL} \cdot \mathrm{ha}^{-1}$ ). O Cd de $1.000 \mathrm{~kg}$, ajustado para $3.890 \mathrm{~kg}=33,26 \mathrm{~kg}$, ou 0,03326 t. Substituindo estes valores na equação obtém-se uma intensidade da doença de $53 \%$ de incidência foliar (Quadro 1) que corresponde a uma perda de $\mathrm{R} \$ 90,00 . \mathrm{ha}^{-1}$. Neste caso o LDE corresponde a uma incidência foliar da ferrugem no estádio de alongamento de $53 \%$.

O princípio de controle integrado de doenças fundamentado no LDE sugere que a aplicação de fungicidas seja realizada no limiar de ação (LA). Portanto, a medida de controle deve ser implementada de modo a evitar que a doença ultrapasse o LDE. Pode-se deduzir que o valor do LDE não é fixo. Por isso, deve ser calculado anualmente e para cada situação em função das variações dos preços da aveia, do fungicida, do custo da aplicação e da eficiência do fungicida.

Com este trabalho concluiu-se que as equações das funções de dano podem ser utilizadas no cálculo do LDE, e que este é uma opção indicadora do momento para proceder-se o controle químico da ferrugem da folha da aveia em cultivares suscetíveis, mantendo a sustentabilidade econômica e ambiental da atividade agrícola.

\section{REFERÊNCIAS BIBLIOGRÁFICAS}

1. Bergamin Filho, A. Importância da quantificação de perdas no manejo de doença de plantas. In: Vale, F.X.R. (Ed.). I Workshop de epidemiologia de doenças de plantas: quantificação de perdas no manejo de doenças de plantas. Viçosa: UFV DFP, 2005. p. 11-16.

2. Forcelini, C.A.; Reis, E.M. Doenças da aveia. In: Kimati, H.; Amorim, L; Rezende, J.A.M.; Bergamin Filho, A.; Camargo, L.E.A. (Ed). Manual de fitopatologia: doenças das plantas cultivadas. 4. ed. São Paulo: Agronômica Ceres, 2005. v.2, p.95-98.

3. Forcelini, C.A.; Reis, E.M.; Floss, E.L.; Berger, R.D.; Camargo, M. In: Reunião da Comissão Sulbrasileira de Pesquisa de Aveia, Reunião da Comissão Brasileira de pesquisa de Aveia, 14. 1994, Porto Alegre Resultados experimentais. Porto Alegre, UFR- 
GS: 1994. p.197-201.

4. Floss, E.L. O papel da aveia como componente de uma agricultura sustentável. In: Reunião da Comissão Brasileira de Pesquisa de Aveia, 21 a , 2001. Resultados Experimentais. Lages, UDESC; 2001. p.11-22.

5. Harder, D.E.; Haber, S. Oat diseases and pathologic techniques. In: Marshall, H.G.; Sorrels, M.E. (Ed.). Oat science and technology. Madison: Editora. 1992. p.307-425 (Agronomy, 33).

6. Indicações técnicas para a cultura da aveia. Passo Fundo: Comissão Brasileira de Pesquisa de Aveia, 2003. 87p.

7. James, W.C. Assessment of plant diseases and losses. Annual Review of Phytopathology, Palo Alto, v.12, p.27-48, 1974.

8. Jesus Junior, W.C.; Vale, F.X.R.; Bergamin Filho, A. Quantificação de danos e perdas. In: Vale, F.X.R.; Jesus Junior, W.C.; Zambolim, L. (Eds). Epidemiologia aplicada ao manejo de doenças de plantas. Belo Horizonte: 2004. p.271-297.

9. Martinelli, J.A. Manejo integrado de doenças da aveia. Fitopatologia Brasileira, Fortaleza, v.28, n.1, p.98-101, 2003

10. Martinelli, J.A.; Federizzi, L.C.; Benneditti, A.C . Redução do rendimento de grãos da aveia em função da severidade da ferrugem da folha. Summa Phytopathologica, Jaguariuna, v.20, n.1, p.116-118, 1994

11. Munford, J.D.; NORTON, G.A. Economics of decision making in pest management. Annual Review of Entomology, Palo Alto, v.29, p.157-174, 1984.
12. Picinini, E.C.; FERNANDES, M.C.; IGNAZAK, J.C.; AMBROSI I. Impacto econômico do uso do fungicida propiconazol na cultura do trigo (Triticum aestivum). Fitopatologia Brasileira, Brasília, v.21, n.3, p.362-368, 1996.

13. Reis, E.M.; Carmona, M. Avaliação do potencial de rendimento de lavouras de trigo com vistas ao controle econômico de doenças foliares com fungicidas. Passo Fundo: UPF. 2001. 28p.

14. Reis, E.M.; Casa, R.T.; Hoffmann, L.L.; Mendes, C.M. Effect of leaf rust on wheat grain yield. Fitopatologia Brasileira, Fortaleza, v.25, n.1, p.67-71, 2000.

15. Reis, E.M.; Casa, R.T.; Medeiros, C.A. Diagnose, patometria e controle de doenças de cereais de inverno. Londrina: ES Comunicação, 2001. 94p.

16. Reis, E.M.; Hoffmann, L.L.; BLUM, M.M. Modelo de ponto crítico para estimar os danos causados pelo oídio em cevada. Fitopatologia Brasileira, Fortaleza, v.27, n.6, p.644-646, 2002.

17. Sah, D.N.; Mackenzie, D.R. Methods of generating different levels of disease epidemics in loss experiments. In: Teng, P.S. (Ed.). Crop loss assessment and pest management. St. Paul, MN: The American Phytopathological Society, 1987. p.90-95.

18. Zadoks, J.C. On the conceptual basis of crop loss assessment: the threshold theory. Annual Review of Phytopathology, Palo Alto, v.23, p.455-473, 1985. 